\title{
Primigravida with history of severe arteriovenous malformations presents for termination
}

\section{Priyanka Debnath, Desiree Fiorentino, Christine L. Curry}

\section{CASE REPORT}

A 23-year-old primigravida presented seeking termination of pregnancy at 11 weeks and 2 days, dated by ultrasound consistent with last menstrual period. It was a medically indicated procedure with significant morbidity due to her past medical history of severe arteriovenous malformations in the liver, portal system, pelvis, and rectum. She had a history of prior venous thrombosis of the gastrointestinal tract, portal hypertension status post shunt placement, and had several dozen treatments of sclerotic therapy and blood transfusions to date. Multiplanar magnetic resonance arteriography and venography with contrast was performed.

An extensive heterogeneous pelvic mass, with a predominantly high T2 signal, had characteristics suggestive of a low-flow malformation (Figure 1). The mass was causing significant mass effect on all pelvic structures (Figure 2), including superior and right sided displacement of the bowel, with displacement of the gravid uterus to the left and out of the pelvis. The mass surrounded adjacent bowel and uterus without direct invasion or cystic characteristics, and it was presumed to be a low-flow malformation without significant arteriovenous shunting.

Based on the severity of the malformations, the patient would not have been able to carry the pregnancy to term without risk of severe bleeding or mortality. The termination and placement of an immediate post-

Priyanka Debnath', Desiree Fiorentino ${ }^{1,2}$, Christine L. Curry ${ }^{1,2}$ Affiliations: 'Department of Obstetrics and Gynecology, University of Miami Miller School of Medicine, Miami, FL, USA; ${ }^{2}$ Jackson Memorial Hospital, Miami, FL, USA.

Corresponding Author: Priyanka Debnath, 170 SE 14th St., \#1105, Miami, FL 33131; Email: pdebnath@miami.edu

Received: 28 November 2015

Accepted: 23 December 2015

Published: 26 February 2016 abortion intrauterine device was completed at 12 weeks and 1 day via ultrasound-guided suction, dilation, and curettage without any intraoperative or postoperative complication.

\section{DISCUSSION}

In pregnancy, arteriovenous malformations (AVMs) sometimes cause an onset of cardiovascular changes such as increase in blood volume and higher blood pressures. The most significant and well-documented risk during pregnancy is due to pulmonary AVMs. There has been no reported increased rate of miscarriage or stillbirths above the population rate [1].

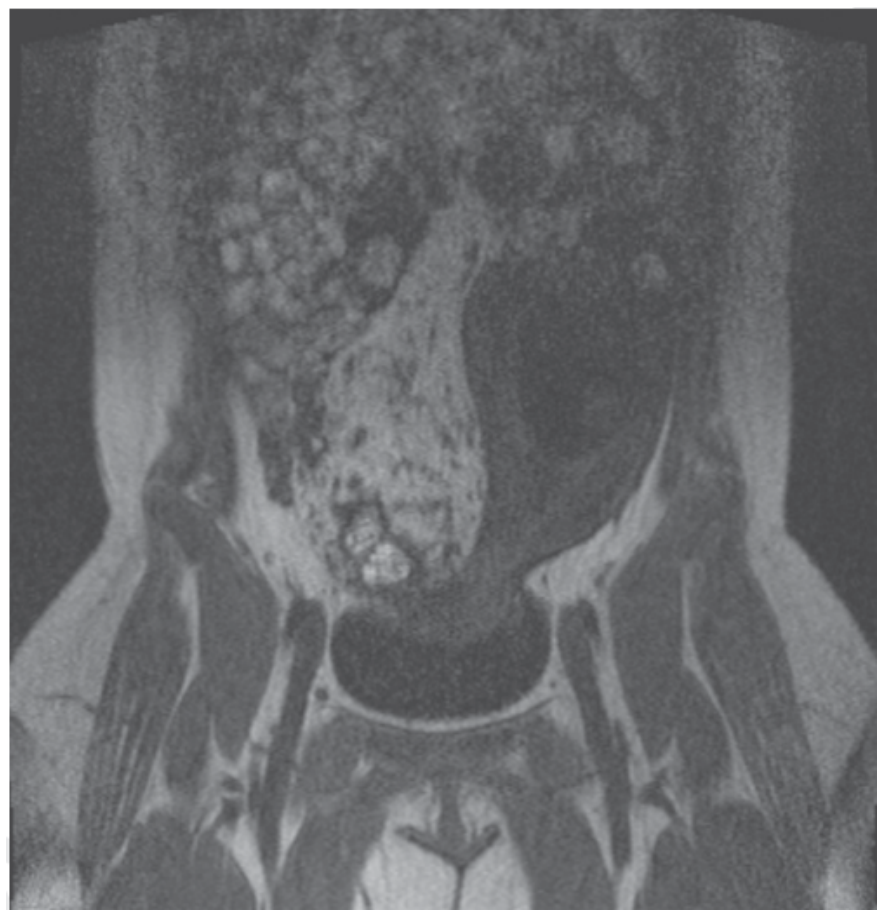

Figure 1: An extensive heterogeneous pelvic mass, with a predominantly high T2 signal, had characteristics suggestive of a low-flow malformation. 


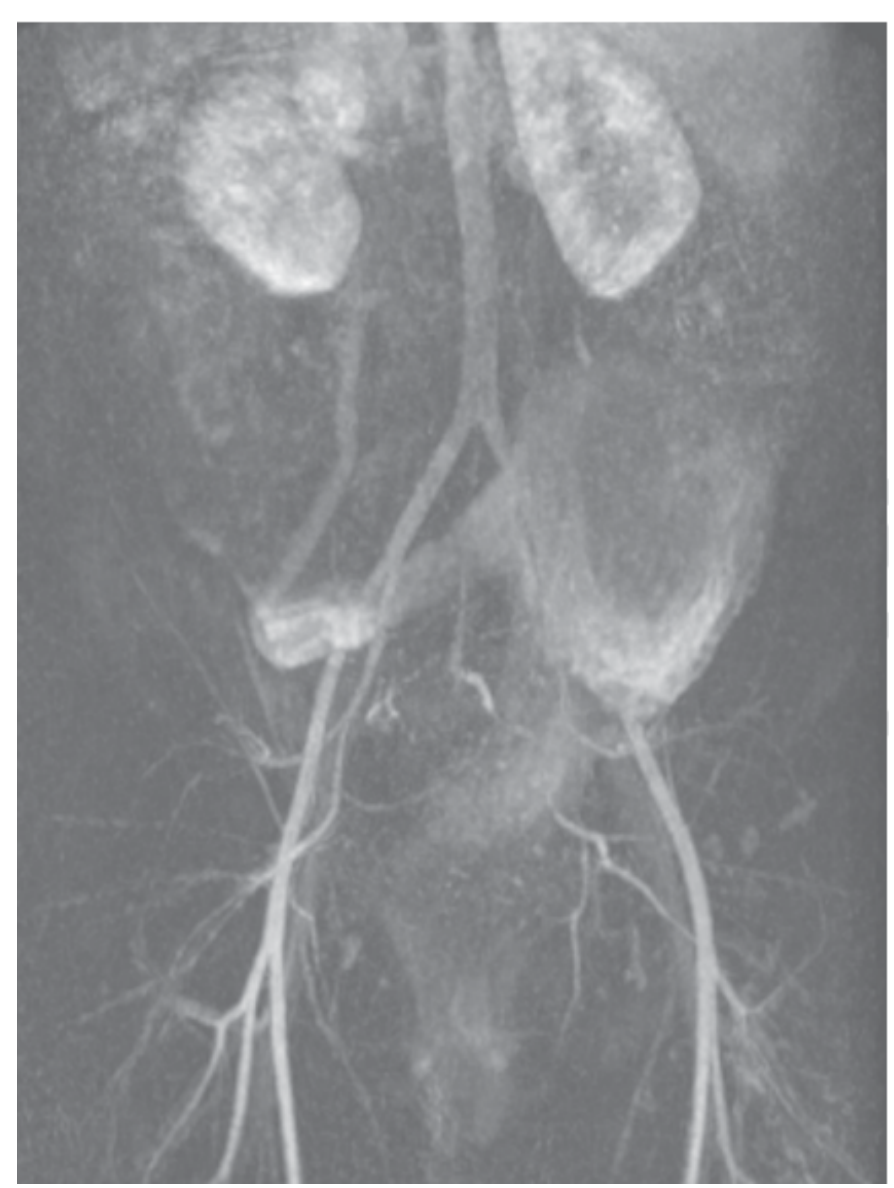

Figure 2: The mass was causing significant mass effect on all pelvic structures and displacement of the cervix and gravid uterus outside of the pelvic brim.

In general, pregnant women in the normal population are found to be at a $3-5 \%$ risk of having a child be born with major congenital malformation or neurologic impairment. In a study of women with Hereditary Hemorrhagic Telangiectasia, there was no increased risk of major congenital malformation, prematurity, cesarean delivery, low-birth weight, or miscarriage compared to the general population [1].

Women with uterine AVMs have a life threatening increased risk of hemorrhage during pregnancy. Weighing risks of intervention against continuation of pregnancy is important based on severity of AVMs, and depends on the clinical status of the patient. Anemic patients should be referred for embolization. Patients who are hemodynamically stable can be treated conservatively [2]. Methods of termination depend on the status of the patient. Though still uncommon, treatments for vascular abnormalities such as embolization and methylergonovine have enabled subsequent successful pregnancies. Spontaneous resolution and successful pregnancy has also been reported [3]. In terms of fertility after vascular uterine abnormalities, studies have shown that fertility is conserved in women with history of these abnormalities, irrespective of management approach [4].

\section{CONCLUSION}

In pregnancy, arteriovenous malformations (AVMs) can cause cardiovascular changes such as increase in blood volume and higher blood pressures which can affect pregnancy outcome.

Keywords: Arteriovenous malformations, Pregnancy, Primigravida, Termination

\section{How to cite this article}

Debnath P, Fiorentino D, Curry CL. Primigravida with history of severe arteriovenous malformations presents for termination. Edorium $\mathrm{J}$ Pathol 2016;2:7-9.

\section{Article ID: 100009Zo8PD2016}

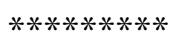

doi:10.5348/Zo8-2016-9-CL-2

$$
* * * * * * * * *
$$

\section{Author Contributions}

Priyanka Debnath - Substantial contributions to conception and design, Acquisition of data, Analysis and interpretation of data, Drafting the article, Revising it critically for important intellectual content, Final approval of the version to be published

Desiree Fiorentino - Substantial contributions to conception and design, Acquisition of data, Analysis and interpretation of data, Drafting the article, Revising it critically for important intellectual content, Final approval of the version to be published

Christine L. Curry - Substantial contributions to conception and design, Acquisition of data, Analysis and interpretation of data, Drafting the article, Revising it critically for important intellectual content, Final approval of the version to be published

\section{Guarantor}

The corresponding author is the guarantor of submission.

\section{Conflict of Interest}

Authors declare no conflict of interest.

\section{Copyright}

(C) 2016 Priyanka Debnath et al. This article is distributed under the terms of Creative Commons Attribution License which permits unrestricted use, distribution and reproduction in any medium provided the original author(s) and original publisher are properly credited. 
Please see the copyright policy on the journal website for more information.

\section{REFERENCES}

1. Wain K, Swanson K, Watson W, Jeavons E, Weaver A, Lindor N. Hereditary hemorrhagic telangiectasia and risks for adverse pregnancy outcomes. Am J Med Genet A 2012 Aug;158A(8):2009-14.

2. O'Brien P, Neyastani A, Buckley AR, Chang SD, Legiehn GM. Uterine arteriovenous malformations: from diagnosis to treatment. J Ultrasound Med 2006 Nov;25(11):1387-92.

3. Castro-Aragon I, Aragon I, Urcuyo R, Abbott J, Levine D. Conservative management of a uterine arteriovenous malformation diagnosed in pregnancy. J Ultrasound Med 2004 Aug;23(8):1101-4.

4. Hugues C, Le Bras Y, Coatleven F, et al. Vascular uterine abnormalities: Comparison of imaging findings and clinical outcomes. Eur J Radiol 2015 Dec;84(12):2485-91.
Access full text article on other devices

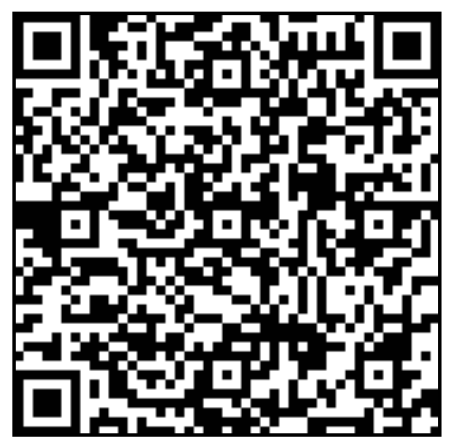

Access PDF of article on other devices

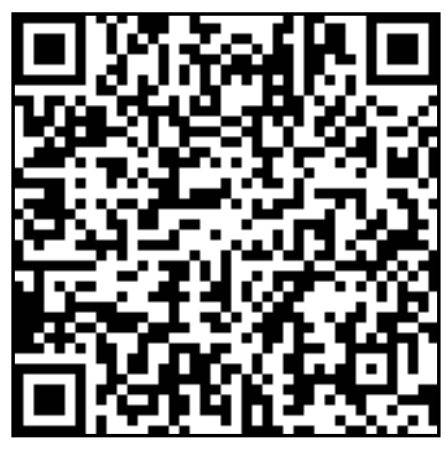

